Check for updates

Cite this: RSC Adv., 2019, 9, 10184

Received 17th February 2019

Accepted 7th March 2019

DOI: 10.1039/c9ra01219b

rsc.li/rsc-advances

\section{Non-alkaloidal constituents from the genus Aconitum: a review}

\begin{abstract}
Tianpeng Yin, ${ }^{\mathrm{ab}}$ Hao Zhou, ${ }^{\mathrm{b}}$ Le Cai ${ }^{\star \mathrm{b}}$ and Zhongtao Ding (D)*b
Species of the genus Aconitum are widely distributed in the north temperate region and have been used in traditional medicine since antiquity due to their biological activities. Phytochemical investigations of Aconitum species have revealed the presence of multiple active ingredients, including flavonoids, phenylpropanoids, phenolics and acids, terpenoids, and polysaccharides in addition to their diterpenoid alkaloids. These non-alkaloidal constituents show great research significance for their novel structures, broad bioactivities, and chemotaxonomical significance. This review addresses, for the first time, the non-alkaloidal constituents and their biological properties and their taxonomic significance for Aconitum plants to facilitate future research.
\end{abstract}

\section{Introduction}

Aconitum L. is a large genus of the Ranunculaceae family comprising approximately 400 species that are distributed mainly in the north temperate region, such as in Asia, Europe, and North America, and more than 200 species of this genus have been found in China. ${ }^{1}$ Plants from this genus have been widely used as herbal medicines in traditional medicine for the treatment of various diseases, such as fainting, rheumatic fever, painful joints, gastroenteritis, diarrhoea, oedema, bronchial asthma, various tumours, and some endocrine disorders, such as irregular menstruation. ${ }^{2,3}$ Especially in China, the first description of the utilization of Aconitum plants in traditional Chinese medicine (TCM) can be traced back to the Han dynasty, approximately two thousand years ago, and it was recorded in the earliest book of Chinese Materia Shennong Bencao Jing. Currently, at least 76 species of Aconitum are used as herbal medicines in China because of their unique and proven therapeutic effects. Several Aconitum-derived herbal drugs, such as Fuzi (processed lateral roots of A. carmichaelii), Chuanwu (processed mother roots of $A$. carmichaelii) and Caowu (processed roots of $A$. kusnezoffii), have become popular and common drugs in TCM. ${ }^{4}$

Aconitum plants are well known for their characteristic diterpenoid alkaloid (DA) components, which have complex chemical structures and remarkable pharmacological activities, especially the prominent anti-inflammatory, antalgic and antiarrhythmic effects. ${ }^{5}$ More than one thousand DAs, which have

${ }^{a}$ Zhuhai Key Laboratory of Fundamental and Applied Research in Traditional Chinese Medicine, Department of Bioengineering, Zunyi Medical University Zhuhai Campus, Zhuhai 519041, China

${ }^{b}$ Functional Molecules Analysis and Biotransformation Key Laboratory of Universities in Yunnan Province, School of Chemical Science and Technology, Yunnan University, Kunming 650091, China.E-mail: ztding@ynu.edu.cn; caile@ynu.edu.cn been structurally classified into four categories, $\mathrm{C}_{18^{-}}, \mathrm{C}_{19^{-}}, \mathrm{C}_{20^{-}}$, and bis-subtypes, have been isolated from this genus. The DAs in Aconitum plants have drawn scientists' lasting attention, and most of the studies on the Aconitum plants were devoted to their DA components. ${ }^{6-8}$ To date, a number of reviews considering various aspects of DAs in Aconitum plants have been published.9,10 Nevertheless, the non-alkaloidal natural constituents in Aconitum plants have also received researchers' strong interests. Recently, various kinds of non-alkaloidal constituents, including flavonoids, phenylpropanoids, phenolics and acids, terpenoids, steroids, free fatty acids (FFAs) and polysaccharides, have been reported to be isolated from the Aconitum plants.

It also shows great significance to study the non-alkaloidal constituents in Aconitum plants. Firstly, the preliminary screening tests showed that the non-alkaloidal constituents from Aconitum plants possess broad and impressive biological activities, including antioxidant, antiparasitic, antiphlogistic, antineoplastic, and immunoregulatory effects, which indicated that these constituents could also serve as a potential medicinal resource for drug discovery. In addition, unlike highly toxic DAs, these non-alkaloidal ingredients are generally less toxic, which is advantageous for the food and pharmaceutical industry. Some kinds of non-alkaloidal constituents from Aconitum plants possess new activities different from DAs, which implied novel potential applications for Aconitum plants in turn. On the other side, it is well known that TCM usually apply a multi-component approach in the treatment of diseases. Certain non-alkaloidal constituents such as phenylpropanoids and polysaccharides that displayed antiphlogistic, antineoplastic and immunore-gulatory effects in accordance with DAs, might also make contribution to the therapeutic effects of Aconitum-derived herbal drugs. Hence, it is beneficial for elucidating the pharmacological material basis and mechanism 
of Aconitum herbs to perform more extensive and in-depth studies on their non-alkaloidal constituents.

Secondly, studies on the non-alkaloidal constituents are conducive to the taxonomy of Aconitum plants. Aconitum is a taxonomically complex genus consisting of many species that are easily confused because their morphological characteristics are indistinguishable. The assistance and supplement from chemotaxonomy is of great importance in the identification of species in Aconitum genus. Research have revealed that, in addition to the DA components, non-alkaloidal constituents for example flavonoids also demonstrated taxonomical significance. These constituents might also be considered as chemotaxonomic markers of this genus to facilitate taxonomy of the Aconitum plants.

Finally, the studies on the non-alkaloidal constituents in Aconitum plants might play a positive role in illuminating the biosynthetic pathway of DAs. A persuasive case was made by the non-alkaloidal diterpenoid atropurpuran from A. hemsleyanum var. atropurpureum, which demonstrated strong relevance in the biosynthetic of hetidine-type DAs. ${ }^{11}$ The studies on the nonalkaloidal constituents might open a new path to explain the construction of some types of DAs whose biosynthetic pathway remains undefined.

Therefore, based on above aspects, this review was conducted to summarize the structural features, biological activities and taxonomical significance of the non-alkaloidal constituents from the Aconitum species for the first time. The aim of this review is to provide a complete overview of the information currently available on the non-alkaloidal constituents of plants from the Aconitum genus, which will facilitate further research and exploitation of this genus.

\section{Non-alkaloidal constituents of the Aconitum genus}

\subsection{Flavonoid glycosides}

Studies on the flavonoid components of Aconitum plants can be traced back to 1969. Russian scientists Samokish et al. first reported the isolation of a flavonoid diglycoside (1) from the leaves of A. orientale (Caucasian monkshood) by paper chromatography. ${ }^{12}$ Flavonoid glycosides are widely distributed in the aerial parts of Aconitum plants, especially in the flowers. However, sample collection is very difficult due to the small size of the petals and the short blooming period, which hinders related research. To date, the flavonoid components of only a few species have been studied, and approximately 55 flavonoid glycosides have been isolated (Fig. 1).

The flavonoids found in Aconitum plants are exclusively glycosides of flavones, with the most common aglycones being kaempferol and quercetin. Compounds $\mathbf{5 4}$ and 55, isolated from A. carmichaelii from Vietnam, are exceptions to this trend, as they possess aglycones of 5,7,4'-tri-methoxylquercetin and 5methoxylluteolin, respectively. ${ }^{13}$ Notably, of the 55 flavonoids presented in Fig. 1, 29 are new glycosides (labelled with *). The novelty of these flavonoid glycosides is determined by the structures of their carbohydrate chains, which are normally located at C-3 or/and C-7, except for compound 55, which has its carbohydrate chain at C-5, a rare substitution pattern. The carbohydrate chain generally appears as a monosaccharide or disaccharide. Among isolated glycosides, new compounds 32, 33, 35, 36, 50, and 51 have trisaccharide chains at C-3. ${ }^{14-16}$ Most of the sugar moieties are glucose, rhamnose, galactose, arabinose or their ethanoyl, benzoyl, caffeoyl, coumaroyl, or feruloyl ester derivatives. New glycoside 19 from A. barbatum and glycoside $\mathbf{5 4}$ from $A$. carmichaeli have rare xylose and fructopyranose substituents, respectively. ${ }^{13,17}$

Natural flavonoid glycosides show broad biological activity, including antioxidant, antibacterial, antiphlogistic and immuno-regulation, antineoplastic, and antiviral effects as well as significant effects on metabolism and on the cardiovascular system of mammals. Among the flavonoid glycosides that have been isolated from Aconitum plants, a series of compounds (1, 16-18, 20-26, 28, 29, 35-38 and 40-45) have demonstrated high antioxidant activity, most of which displayed DPPH radical scavenging activity. ${ }^{15,18,19}$ In particular, glycosides 20 and 42 exhibited impressive antiradical activities $\left(\mathrm{IC}_{50}\right.$ values of 1.9 and $2.0 \mu \mathrm{M}$, respectively), and they were more potent than the positive controls, quercetin and rutin $\left(\mathrm{IC}_{50}\right.$ values of 2.5 and 3.9 $\mu \mathrm{M}$, respectively). Quercetin glycosides possess better antioxidant activities than kaempferol glycosides, and the caffeoyl derivatives have a higher radical scavenging abilities than the $p$ coumaroyl esters, which could be attributed to the vital effect of the ortho-dihydroxy groups in their structures. ${ }^{20}$ Since most of DAs are ineffective in scavenging radicals, ${ }^{21,22}$ and the content of DAs in the flowers of Aconitum plants is pretty low, it suggests that flavonoid glycosides could be responsible for the antioxidant activities of the Aconitum flowers. ${ }^{15}$

Moreover, new compounds 23 and 24, isolated from $A$. napellus subsp. lusitanicum, along with other flavonoid glycosides from several Delphinium species, were tested for their antiparasitic activities against three Leishmania spp. and Trypanosoma cruzi. ${ }^{23}$ Compound 23 showed a higher trypanocidal activity with an $\mathrm{IC}_{50}$ value of $11.0 \mu \mathrm{M}$ against the intracellular form of T. cruzi than was observed with the positive control BZN, which exerted an $\mathrm{IC}_{50}$ value of $23.3 \mu \mathrm{M}$. Compound 24 showed higher leishmanicidal activities against both the extra- and intracellular forms of $L$. infantum $\left(\mathrm{IC}_{50}\right.$ values of 16.7 and 12.8 $\mu \mathrm{M}$, respectively) and $L$. donovani $\left(\mathrm{IC}_{50}\right.$ values of 12.5 and 15.5 $\mu \mathrm{M}$, respectively) than the reference drug glucantime. Notably, compounds 23 and 24 also showed less toxicity to the corresponding host cells, highlighting their potential in the treatment of leishmaniasis and Chagas disease. Normally, the roots are the only officinal parts of Aconitum plants. The reseach presented above suggest that the flowers with abundant bioactive flavonoid glycosides might serve as a new officinal part of the Aconitum plants.

On the other side, similar to DAs, flavonoid components have also been proposed as chemotaxonomic markers of Aconitum plants to facilitate taxonomic discrimination. Fico et al. demonstrated that flavonoid profiles can be used to differentiate two A. napellus subspecies and that in turn, their flavonoid profiles differed from the profiles of A. paniculatum and A. vulparia. As a result, they suggested that species recognition within 


\begin{tabular}{|c|c|c|c|}
\hline No & $R_{1}, R_{2}, R_{3}, R_{4}$ & plant & ref \\
\hline \multirow[t]{2}{*}{1} & $\mathrm{R}_{1}=\mathrm{R}_{3}=O-\alpha-\mathrm{L}-\mathrm{Rha}, \mathrm{R}_{2}=\mathrm{OH}, \mathrm{R}_{4}=\mathrm{H}$ & A. orientale & 12 \\
\hline & & A. vulparis & 27 \\
\hline 2 & $\mathrm{R}_{1}=\mathrm{R}_{3}=O-\mathrm{B}_{-\mathrm{D}}-\mathrm{Glu}, \mathrm{R}_{2}=\mathrm{OH}, \mathrm{R}_{4}=\mathrm{H}$ & A. orientale & 28 \\
\hline 3 & $\mathrm{R}_{1}=\mathrm{R}_{3}=\mathrm{O}-\mathrm{B}-\mathrm{D}-\mathrm{G} l \mathrm{u}, \mathrm{R}_{2}=\mathrm{R}_{4}=\mathrm{OH}$ & A. orientale & 28 \\
\hline 4 & $\mathrm{R}_{3}=\mathrm{O}-\alpha-\mathrm{L}-\mathrm{Rha}, \mathrm{R}_{1}=\mathrm{R}_{2}=\mathrm{OH}, \mathrm{R}_{4}=\mathrm{H}$ & A. baicalense & 29 \\
\hline \multirow[t]{2}{*}{5} & $\mathrm{R}_{3}=O-\alpha-\mathrm{L}-\mathrm{Rha}, \mathrm{R}_{1}=\mathrm{R}_{2}=\mathrm{R}_{4}=\mathrm{OH}$ & A. baicalense & 29 \\
\hline & & A. vulparis & 27 \\
\hline $6^{*}$ & $\mathrm{R}_{1}=O-[6-E$-caffeoyl- $6-\mathrm{D}-\mathrm{Glu}-(1 \rightarrow 2)-6-\mathrm{D}-\mathrm{Glu}], \mathrm{R}_{3}=O-\alpha-\mathrm{L}-\mathrm{Rha}, \mathrm{R}_{2}=\mathrm{R}_{4}=\mathrm{OH}$ & A. baicalense & 29 \\
\hline 7 & $\mathrm{R}_{1}=O-B-\mathrm{D}-\mathrm{Gal}, \mathrm{R}_{2}=\mathrm{R}_{3}=\mathrm{R}_{4}=\mathrm{OH}$ & A. jaluense for. album & 30 \\
\hline \multirow[t]{2}{*}{8} & $\mathrm{R}_{1}=O-B-\mathrm{D}-\mathrm{Gal}, \mathrm{R}_{2}=\mathrm{R}_{3}=\mathrm{OH}, \mathrm{R}_{4}=\mathrm{H}$ & A. jaluense for. album & 30 \\
\hline & & $\begin{array}{l}\text { A. pseudolaeve var. } \\
\text { erectum }\end{array}$ & 31 \\
\hline 9 & $\mathrm{R}_{1}=O-[6-\mathrm{D}-\mathrm{Gal}-(1 \rightarrow 6)-6-\mathrm{D}-\mathrm{Glu}], \mathrm{R}_{2}=\mathrm{R}_{3}=\mathrm{OH}, \mathrm{R}_{4}=\mathrm{H}$ & A. jaluense for. album & 30 \\
\hline $10^{*}$ & $\mathrm{R}_{1}=O-B_{-\mathrm{D}}-\mathrm{Glu}, \mathrm{R}_{3}=O-\left[\left(6-E\right.\right.$-caffeoyl)- $\left.{ }_{-}-\mathrm{D}-\mathrm{Glu}-(1 \rightarrow 2)-\alpha-\mathrm{L}-\mathrm{Rha}\right], \mathrm{R}_{2}=\mathrm{OH}, \mathrm{R}_{4}=\mathrm{H}$ & A. chilsanense & 32 \\
\hline $11^{*}$ & $\mathrm{R}_{1}=O-B-\mathrm{D}-\mathrm{Glu}, \mathrm{R}_{3}=\mathrm{O}-\left[6-E\right.$-caffeoyl- 6 -D-Glu- $\left.(1 \rightarrow 2)-\alpha-{ }_{-}-\mathrm{Rha}\right], \mathrm{R}_{2}=\mathrm{R}_{4}=\mathrm{OH}$ & A. chilsanense & 32 \\
\hline $12^{*}$ & $\mathrm{R}_{1}=O--_{-\mathrm{D}-\mathrm{Glu}}, \mathrm{R}_{3}=O-\left[6-E\right.$-feruloyl- $\left.-{ }_{-\mathrm{D}-\mathrm{G}} \mathrm{Glu}-(1 \rightarrow 2)-\alpha-\mathrm{L}_{-}-\mathrm{Rha}\right], \mathrm{R}_{2}=\mathrm{OH}, \mathrm{R}_{4}=\mathrm{H}$ & A. chilsanense & 32 \\
\hline 13* & $\mathrm{R}_{1}=O-B-\mathrm{D}-\mathrm{Glu}, \mathrm{R}_{3}=O-\left(6\right.$-benzoyl)- $-\mathrm{-G}$-u- $(1 \rightarrow 2)-\alpha-\mathrm{L}-\mathrm{Rha}, \mathrm{R}_{2}=\mathrm{OH}, \mathrm{R}_{4}=\mathrm{H}$ & A. chilsanense & 32 \\
\hline $14 *$ & $\mathrm{R}_{3}=O-[6-E$-caffeoyl- $-\mathrm{-D}-\mathrm{Glu}-(1 \rightarrow 2)-\alpha-\mathrm{L}-\mathrm{Rha}], \mathrm{R}_{1}=\mathrm{R}_{2}=\mathrm{OH}, \mathrm{R}_{4}=\mathrm{H}$ & A. chilsanense & 32 \\
\hline $15^{*}$ & $\mathrm{R}_{3}=O-[6-\mathrm{D}-\mathrm{Gal}-(1 \rightarrow 2)-\alpha-\mathrm{L}-\mathrm{Rha}], \mathrm{R}_{1}=\mathrm{R}_{2}=\mathrm{OH}, \mathrm{R}_{4}=\mathrm{H}$ & A. chilsanense & 32 \\
\hline $16 *$ & $\mathrm{R}_{1}=O-\left(2^{\prime \prime}-\right.$ acetyl- $\left.-6-\mathrm{D}-\mathrm{Gal}\right), \mathrm{R}_{2}=\mathrm{R}_{3}=\mathrm{OH}, \mathrm{R}_{4}=\mathrm{H}$ & A. paniculatum & 33 \\
\hline $17^{*}$ & $\mathrm{R}_{1}=O-\left(2^{\prime \prime}\right.$-acetyl- 6 -D-Gal), $\mathrm{R}_{3}=O-\alpha-\mathrm{L}-\mathrm{Ara}, \mathrm{R}_{2}=\mathrm{OH}, \mathrm{R}_{4}=\mathrm{H}$ & A. paniculatum & 33 \\
\hline \multirow[t]{2}{*}{ 18* } & $\mathrm{R}_{1}=O-\left(2^{\prime \prime}\right.$-acetyl- 6 - $\left.\mathrm{D}-\mathrm{Gal}\right), \mathrm{R}_{3}=O-\alpha-\mathrm{L}-\mathrm{Ara}, \mathrm{R}_{2}=\mathrm{R}_{4}=\mathrm{OH}$ & A. paniculatum & 33 \\
\hline & & A. angustifolium & 34 \\
\hline $19 *$ & $\mathrm{R}_{1}=O-\left[3,4-\right.$ di-O-acetyl- - -Xyl- $\alpha$-Rha], $\mathrm{R}_{3}=O-\alpha-\mathrm{-L}-\mathrm{Rha}, \mathrm{R}_{2}=\mathrm{OH}, \mathrm{R}_{4}=\mathrm{H}$ & A. barbatum & 17 \\
\hline \multirow[t]{3}{*}{$20^{*}$} & $\mathrm{R}_{1}=O-B-\mathrm{D}-\mathrm{Glu}, \mathrm{R}_{3}=O-[6-E-$ caffeoyl- - -D-Glu- $(1 \rightarrow 3)-\alpha-\mathrm{-}-\mathrm{Rha}], \mathrm{R}_{2}=\mathrm{R}_{4}=\mathrm{OH}$ & A. napellus ssp. & 35 \\
\hline & & neomontanum & 34 \\
\hline & & A. angustifolium & \\
\hline \multirow[t]{3}{*}{ 21* } & $\mathrm{R}_{1}=O-6-\mathrm{D}-\mathrm{Glu}, \mathrm{R}_{3}=O-\left[6-E-\right.$ caffeoyl- $\left.6-\mathrm{o}_{-}-\mathrm{Glu}-(1 \rightarrow 3)-\alpha-\mathrm{L}-\mathrm{Rha}\right], \mathrm{R}_{2}=\mathrm{OH}, \mathrm{R}_{4}=\mathrm{H}$ & A. napellus ssp. & 35 \\
\hline & & neomontanum & 34 \\
\hline & & A. angustifolium & \\
\hline \multirow[t]{3}{*}{$22^{*}$} & $\mathrm{R}_{1}=O-B-\mathrm{D}-\mathrm{Glu}, \mathrm{R}_{3}=O-[6-E-p$-coumaroyl- $-6-\mathrm{D}-\mathrm{Glu}-(1 \rightarrow 3)-\alpha-\mathrm{L}-\mathrm{Rha}], \mathrm{R}_{2}=\mathrm{OH}, \mathrm{R}_{4}=\mathrm{H}$ & A. napellus ssp. & 35 \\
\hline & & neomontanum & 34 \\
\hline & & A. angustifolium & \\
\hline \multirow[t]{3}{*}{ 23* } & $\mathrm{R}_{1}=O-[6-E-$ caffeoyl- $-6-\mathrm{D}-\mathrm{Glu}-(1 \rightarrow 2)-6-\mathrm{D}-\mathrm{Glu}], \mathrm{R}_{3}=O-\alpha-\mathrm{L}-\mathrm{Rha}, \mathrm{R}_{2}=\mathrm{R}_{4}=\mathrm{OH}$ & A. napellus ssp. tauricum & 36 \\
\hline & & A. napellus subsp. & 25 \\
\hline & & lusitanicum & \\
\hline 24* & $\mathrm{R}_{1}=O-\left[6-E-\right.$ caffeoyl- $\left.-{ }_{-\mathrm{D}}-\mathrm{Glu}-(1 \rightarrow 2)-{ }_{-\mathrm{D}-\mathrm{G}} \mathrm{G}\right], \mathrm{R}_{3}=O-\alpha-\mathrm{L}-\mathrm{Rha}, \mathrm{R}_{2}=\mathrm{OH}, \mathrm{R}_{4}=\mathrm{H}$ & A. napellus ssp. tauricum & 36 \\
\hline
\end{tabular}

Fig. 1 Flavonoid glycosides from the Aconitum plants. 


\begin{tabular}{|c|c|c|c|}
\hline $25^{*}$ & $\mathrm{R}_{1}=O-\left[6-E-p\right.$-coumaroyl- $\left.6{ }_{-\mathrm{D}}-\mathrm{Glu}-(1 \rightarrow 2)-6-\mathrm{D}-\mathrm{Glu}\right], \mathrm{R}_{3}=O-\alpha-\mathrm{L}-\mathrm{Rha}, \mathrm{R}_{2}=\mathrm{R}_{4}=\mathrm{OH}$ & $\begin{array}{l}\text { A. napellus ssp. tauricum } \\
\text { A. napellus subsp. } \\
\text { lusitanicum }\end{array}$ & 25 \\
\hline 26* & $\mathrm{R}_{1}=O-\left[6-E-p\right.$-coumaroyl- $\left.-{ }_{-\mathrm{D}}-\mathrm{Glu}-(1 \rightarrow 2)-{ }_{-\mathrm{D}}-\mathrm{Glu}\right], \mathrm{R}_{3}=O-\alpha-\mathrm{L}-\mathrm{Rha}, \mathrm{R}_{2}=\mathrm{OH}, \mathrm{R}_{4}=\mathrm{H}$ & A. napellus ssp. tauricum & 36 \\
\hline 27 & $\mathrm{R}_{1}=O-B-\mathrm{D}-\mathrm{Gal}, \mathrm{R}_{3}=O-\alpha-\mathrm{L}-\mathrm{Rha}, \mathrm{R}_{2}=\mathrm{R}_{4}=\mathrm{OH}$ & A. vulparis & 27 \\
\hline 28 & $\mathrm{R}_{1}=O-\theta-\mathrm{D}-\mathrm{Glu}, \mathrm{R}_{3}=O-\alpha-\mathrm{L}-\mathrm{Rha}, \mathrm{R}_{2}=\mathrm{OH}, \mathrm{R}_{4}=\mathrm{H}$ & $\begin{array}{l}\text { A. vulparis } \\
\text { A. naviculare }\end{array}$ & 27 \\
\hline 29 & $\mathrm{R}_{1}=\mathrm{R}_{3}=O-\alpha-\mathrm{L}-\mathrm{Rha}, \mathrm{R}_{2}=\mathrm{R}_{4}=\mathrm{OH}$ & A. vulparis & 27 \\
\hline 30 & $\mathrm{R}_{1}=O-B-\mathrm{D}-\mathrm{Glu}, \mathrm{R}_{3}=O-\alpha-\mathrm{L}-\mathrm{Rha}, \mathrm{R}_{2}=\mathrm{R}_{4}=\mathrm{OH}$ & $\begin{array}{l}\text { A. napellus subsp. } \\
\text { lusitanicum }\end{array}$ & 25 \\
\hline 31 & $\mathrm{R}_{1}=O-[6-\mathrm{D}-\mathrm{Glu}-(1 \rightarrow 2)-6-\mathrm{D}-\mathrm{Glu}], \mathrm{R}_{3}=O-\alpha-\mathrm{L}-\mathrm{Rha}, \mathrm{R}_{2}=\mathrm{R}_{4}=\mathrm{OH}$ & A. napellus sp. Iusitanicum & 38 \\
\hline $32 *$ & $\begin{array}{l}\mathrm{R}_{1}=O-\left[6-\mathrm{D}-\mathrm{Glu}-(1 \rightarrow 3)-(4-\mathrm{O}-\mathrm{E}-\mathrm{p}-\text { coumaroyl)- } \alpha-\mathrm{L}-\mathrm{Rha}-(1 \rightarrow 6)-6-\mathrm{D}-\mathrm{Glu}], \mathrm{R}_{3}=0-\left[6-\mathrm{D}-\mathrm{Glu}-(1 \rightarrow 3)-\alpha-\mathrm{L}^{-}\right.\right. \\
\mathrm{Rha}], \mathrm{R}_{2}=\mathrm{OH}, \mathrm{R}_{4}=\mathrm{H}\end{array}$ & A. naviculare & 14 \\
\hline $33^{*}$ & $\begin{array}{l}\mathrm{R}_{1}=O-\left[6-{ }_{-}-\mathrm{Glu}-(1 \rightarrow 3)-\left(4-O-E-p-\text { coumaroyl)- } \alpha-{ }_{\mathrm{L}}-\mathrm{Rha}-(1 \rightarrow 6)-6{ }_{\mathrm{D}}-\mathrm{Glu}\right], \mathrm{R}_{3}=O-\left[6-\mathrm{D}-\mathrm{Glu}-(1 \rightarrow 3)-\alpha_{-\mathrm{L}^{-}}\right.\right. \\
\mathrm{Rha}], \mathrm{R}_{2}=\mathrm{R}_{4}=\mathrm{OH}\end{array}$ & A. naviculare & 14 \\
\hline $34^{*}$ & $\mathrm{R}_{3}=O-\left[B_{-\mathrm{D}}-\mathrm{Glu}-(1 \rightarrow 3)-\alpha-{ }_{-}-\mathrm{Rh} a\right], \mathrm{R}_{1}=\mathrm{R}_{2}=\mathrm{R}_{4}=\mathrm{OH}$ & A. naviculare & 14 \\
\hline $35^{*}$ & $\begin{array}{l}\mathrm{R}_{1}=O-\left[6-\mathrm{D}-\mathrm{Glu}-(1 \rightarrow 3)-\left(4-O-(E-p-\text { coumaroyl })-\alpha-{ }_{-}-\mathrm{Rha}-(1 \rightarrow 6)-6-\mathrm{D}-\mathrm{Gal}\right], \mathrm{R}_{3}=O-\alpha-\mathrm{L}-\mathrm{Rha}, \mathrm{R}_{2}=\mathrm{R}_{4}=\right. \\
\mathrm{OH}\end{array}$ & A. anthora & 15 \\
\hline $36 *$ & $\begin{array}{l}\mathrm{R}_{1}=O-\left[6-\mathrm{D}-\mathrm{Glu}-(1 \rightarrow 3)-(4-O-(E-p-\text { coumaroyl })-\alpha-\mathrm{L}-\mathrm{Rha}-(1 \rightarrow 6)-6-\mathrm{D}-\mathrm{Gal}], \mathrm{R}_{3}=O-\alpha-\mathrm{L}-\mathrm{Rh}, \mathrm{R}_{2}=\mathrm{OH}, \mathrm{R}_{4}\right. \\
=\mathrm{H}\end{array}$ & A. anthora & 15 \\
\hline 37 & $\left.\mathrm{R}_{1}=O-[\alpha-\mathrm{L}-\mathrm{Rha}-(1 \rightarrow 6)-6-\mathrm{D}-\mathrm{Ga}]\right], \mathrm{R}_{3}=O-\alpha-\mathrm{L}-\mathrm{Rha}, \mathrm{R}_{2}=\mathrm{R}_{4}=\mathrm{OH}$ & A. anthora & 15 \\
\hline 38 & $\mathrm{R}_{1}=O-\left[\alpha-\mathrm{L}-\mathrm{Rha}-(1 \rightarrow 6)-6-\mathrm{D}-\mathrm{Ga}\right.$ l] $, \mathrm{R}_{3}=\mathrm{O}-\alpha-\mathrm{L}-\mathrm{Rha}, \mathrm{R}_{2}=\mathrm{OH}, \mathrm{R}_{4}=\mathrm{H}$ & A. anthora & 15 \\
\hline 39 & $\mathrm{R}_{3}=O-\left[B_{-\mathrm{D}}-\mathrm{Glu}(1 \rightarrow 3)-\alpha-\mathrm{L}-\mathrm{Rha}\right], \mathrm{R}_{1}=\mathrm{R}_{2}=\mathrm{OH}, \mathrm{R}_{4}=\mathrm{H}$ & A. naviculare & 37 \\
\hline $40^{*}$ & $\mathrm{R}_{1}=O-6-\mathrm{D}-\mathrm{Glu}, \mathrm{R}_{3}=O-[6-E-p$-coumaroyl- $-\mathrm{-D}-\mathrm{Glu}-(1 \rightarrow 3)-\alpha-\mathrm{L}-\mathrm{Rha}], \mathrm{R}_{2}=\mathrm{R}_{4}=\mathrm{OH}$ & A. burnatii & 19 \\
\hline $41^{*}$ & $\mathrm{R}_{1}=O-6-\mathrm{D}-\mathrm{Glu}, \mathrm{R}_{3}=O-[6-\mathrm{D}-\mathrm{Glu}-(1 \rightarrow 3)-\alpha-\mathrm{-}-\mathrm{Rh} a], \mathrm{R}_{2}=\mathrm{R}_{4}=\mathrm{OH}$ & $\begin{array}{l}\text { A. burnatii } \\
\text { A. angustifolium }\end{array}$ & 19 \\
\hline 42 & $\mathrm{R}_{1}=O-6-\mathrm{D}-\mathrm{Glu}, \mathrm{R}_{3}=O-\left[6-E-\right.$ caffeoyl- $\left.--_{-\mathrm{D}}-\mathrm{Glu}-(1 \rightarrow 3)-\alpha-\mathrm{L}-\mathrm{Rha}\right], \mathrm{R}_{2}=\mathrm{R}_{4}=\mathrm{OH}$ & A. burnatii & 19 \\
\hline 43* & $\mathrm{R}_{1}=O-6-\mathrm{D}-\mathrm{Glu}, \mathrm{R}_{3}=O-\alpha-\mathrm{L}-\mathrm{Ara}, \mathrm{R}_{2}=\mathrm{OH}, \mathrm{R}_{4}=\mathrm{H}$ & A. variegatum & 19 \\
\hline 44 & $\mathrm{R}_{1}=O--_{-\mathrm{D}}-\mathrm{Glu}, \mathrm{R}_{2}=\mathrm{R}_{3}=\mathrm{R}_{4}=\mathrm{OH}$ & A. variegatum & 19 \\
\hline 45 & $\mathrm{R}_{1}=O-6-\mathrm{D}-\mathrm{Glu}, \mathrm{R}_{2}=\mathrm{R}_{3}=\mathrm{OH}, \mathrm{R}_{4}=\mathrm{H}$ & A. variegatum & 19 \\
\hline $46 *$ & $\mathrm{R}_{1}=O-\theta_{-\mathrm{D}}-\mathrm{Gal}, \mathrm{R}_{3}=O-[6-E-p$-coumaroyl- $6-\mathrm{D}-\mathrm{Glu}-(1 \rightarrow 3)-\alpha-\mathrm{L}-\mathrm{Rha}], \mathrm{R}_{2}=\mathrm{OH}, \mathrm{R}_{4}=\mathrm{H}$ & A. angustifolium & 34 \\
\hline 47 & $\mathrm{R}_{1}=O-\left[\alpha-\mathrm{L}-\mathrm{Rha}-(1 \rightarrow 6)-\mathrm{-}_{-\mathrm{D}}-\mathrm{Ga} a \mathrm{l}\right], \mathrm{R}_{3}=\alpha-\mathrm{L}-\mathrm{Rha}, \mathrm{R}_{2}=\mathrm{OH}, \mathrm{R}_{4}=\mathrm{H}$ & A. tanguticum & 39 \\
\hline 48 & $\mathrm{R}_{1}=O-\left[\alpha-\mathrm{L}-\mathrm{Rha}-(1 \rightarrow 6)-{ }_{-\mathrm{D}-\mathrm{-G}} \mathrm{u}\right], \mathrm{R}_{3}=\alpha-\mathrm{L}-\mathrm{Rha}, \mathrm{R}_{2}=\mathrm{OH}, \mathrm{R}_{4}=\mathrm{H}$ & A. tanguticum & 39 \\
\hline 49 & $\mathrm{R}_{3}=O-\alpha-\mathrm{L}-\mathrm{Rha}, \mathrm{R}_{1}=\mathrm{R}_{2}=\mathrm{OH}, \mathrm{R}_{4}=\mathrm{H}$ & A. tanguticum & 39 \\
\hline $50 *$ & $\begin{array}{l}\mathrm{R}_{1}=O-[\alpha-\mathrm{L}-\mathrm{Rha}-(1 \rightarrow 2)-[6-\mathrm{D}-\mathrm{Glu}-(1 \rightarrow 3)-\alpha-\mathrm{L}-(4-E-p-\text { coumaroyl-Rha })-(1 \rightarrow 6)]-6-\mathrm{D}-\mathrm{Gal}], \mathrm{R}_{3}=O-\alpha-\mathrm{-}- \\
\text { rha, } \mathrm{R}_{2}=\mathrm{R}_{4}=\mathrm{OH}\end{array}$ & A. tanguticum & 16 \\
\hline $51 *$ & 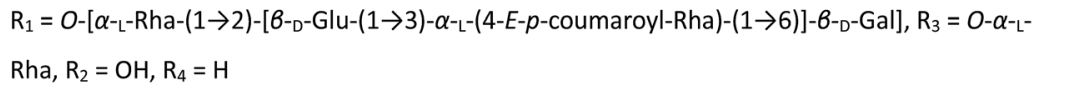 & A. tanguticum & 16 \\
\hline 52 & $\mathrm{R}_{1}=O-[\alpha-\mathrm{L}-\mathrm{Rha}-(1 \rightarrow 2)-\alpha-\mathrm{L}-\mathrm{Rha}-(1 \rightarrow 6)-6-\mathrm{D}-\mathrm{Gal}], \mathrm{R}_{3}=0-\alpha-\mathrm{-}-\mathrm{Rha}, \mathrm{R}_{2}=\mathrm{R}_{4}=\mathrm{OH}$ (quercetin) & A. tanguticum & 16 \\
\hline 53 & $\mathrm{R}_{1}=O-\left[\alpha-\mathrm{L}-\mathrm{Rha}-(1 \rightarrow 2)-\alpha-\mathrm{L}-\mathrm{Rha}-(1 \rightarrow 6)-\beta_{-\mathrm{D}}-\mathrm{Gal}\right], \mathrm{R}_{3}=O-\alpha-\mathrm{L}-\mathrm{Rha}, \mathrm{R}_{2}=\mathrm{OH}, \mathrm{R}_{4}=\mathrm{H}$ & A. tanguticum & 16 \\
\hline 54 & $\mathrm{R}_{1}=O-B_{-\mathrm{D}}-\mathrm{Fru}, \mathrm{R}_{2}=\mathrm{R}_{3}=\mathrm{R}_{4}=\mathrm{OMe}$ & A. carmichaelii & 13 \\
\hline 55 & 4'-OMe, $\mathrm{R}_{2}=O-\left[\alpha-\llcorner-\mathrm{Ara}-(1 \rightarrow 6)-\beta-\mathrm{D}-\mathrm{Glu}], \mathrm{R}_{1}=\mathrm{H}, \mathrm{R}_{3}=\mathrm{OMe}, \mathrm{R}_{4}=\mathrm{OH}\right.$ & A. carmichaelii & 13 \\
\hline
\end{tabular}

Fig. 1 (contd.) 


\begin{tabular}{lllll} 
& & & \\
& & & & \\
\hline No & name & $\mathrm{R}_{1}, \mathrm{R}_{2}, \mathrm{R}_{3}, \mathrm{R}_{4}$ & plnats & ref. \\
\hline $\mathbf{5 7}$ & 4,5 -dicaffeoylquinic acid & $\mathrm{R}_{1}=\mathrm{R}_{2}=\mathrm{H}, \mathrm{R}_{3}=\mathrm{R}_{4}=$ caffeoyl & A. koreanum & 44 \\
$\mathbf{5 8}$ & 3,5 -dicaffeoylquinic acid & $\mathrm{R}_{1}=\mathrm{R}_{3}=\mathrm{H}, \mathrm{R}_{2}=\mathrm{R}_{4}=$ caffeoyl & A. koreanum & 44 \\
$\mathbf{5 9}$ & 3,5-dicaffeoylquinic acid methyl ester & $\mathrm{R}_{3}=\mathrm{H}, \mathrm{R}_{1}=\mathrm{Me}, \mathrm{R}_{2}=\mathrm{R}_{4}=$ caffeoyl & A. koreanum & 44 \\
\hline
\end{tabular}

\begin{tabular}{lllll} 
& & & \\
\hline No & name & $\mathrm{R}_{1}, \mathrm{R}_{2}, \mathrm{R}_{3}$ & plants & ref. \\
\hline $\mathbf{6 1 *}$ & $(Z)$-sinapic acid-4-O-B-D-allopyranoside & $\mathrm{R}_{1}=\mathrm{O}-\mathrm{B}-\mathrm{D}-\mathrm{All}, \mathrm{R}_{2}=\mathrm{R}_{3}=\mathrm{OMe}$ & A. tanguticum & 40 \\
$\mathbf{8 1}$ & $(Z)$ - $p$-hydroxycinnamic acid & $\mathrm{R}_{1}=\mathrm{OH}, \mathrm{R}_{2}=\mathrm{R}_{3}=\mathrm{H}$ & A. stapfianum & 45 \\
\hline
\end{tabular}

\begin{tabular}{|c|c|c|c|c|}
\hline No & name & $\mathrm{R}_{1}, \mathrm{R}_{2}$ & plants & ref. \\
\hline $62^{*}$ & 3,4-dihydroxyphenethoxy-8-O-B-D-[6-O-(4-O-B-D-glucopyranosyl)-feruloyl]-glucopyranoside & $\mathrm{R}_{1}=\mathrm{OH}, \mathrm{R}_{2}=\mathrm{H}$ & A. tanguticum & 40 \\
\hline 63 & 4-dihydroxyphenethoxy-8-O-6-o-[6-O-(4-O-B-D-glucopyranosyl)-feruloyl]-glucopyranoside & $\mathrm{R}_{1}=\mathrm{R}_{2}=\mathrm{H}$ & A. tanguticum & 40 \\
\hline 64* & 4-hydroxyphenethoxy-8-O- 6 -o-[6-O-(4-O- - -D-glucopyranosyl)-sinapoyl]-glucopyranoside & $\mathrm{R}_{1}=\mathrm{H}, \mathrm{R}_{2}=\mathrm{OMe}$ & A. tanguticum & 41 \\
\hline
\end{tabular}

Fig. 2 Phenylpropionic acids from Aconitum plants.

this large genus can be approached by using flavonoids as chemical molecular markers. ${ }^{24,25} \operatorname{Lim}$ et al. also proved the utility of flavonoid analysis for the chemotaxonomy of Aconitum in their study on the flavonoid variations in members of the Korean A. jaluense complex.. ${ }^{26}$ However, the relationship between the flavonoid composition and the degree of evolution of the Aconitum species remains unclear and requires more extensive and in-depth studies. Flavonoids and DAs are all common constituents in Aconitum plants distributed in different parts. Hence, we suggest that the chemotaxonomical studies on Aconitum plants should consider the flavonoid and DA components together, through analysis not only the structural diversity of flavonoid and DA components severally, but also their correlations in taxonomy of Aconitum genus.

\subsection{Phenylpropanoids}

Phenylpropanoids, perhaps the most widespread type of natural products found in the plant kingdom, are composed of $\mathrm{C}_{6}-\mathrm{C}_{3}$ structural units biosynthesized from shikimic acid. Phenylpropanoids can be divided into three categories: phenylpropionic acids, coumarins and lignans. A series of phenylpropanoids isolated from Aconitum plants have been reported.

As shown in Fig. 2, 22 phenylpropionic acids, including 6 new compounds (labelled with ${ }^{*}$ ), have been isolated from 8 species of the Aconitum genus. ${ }^{40-42}$ Phenylpropionic acids from Aconitum plants are generally glycosides of caffeic acid (56), ferulic acid (76), or $p$-hydroxycinnamic acid (80). Most of the double bonds in those acids are in the trans form. (Z)-Sinapic acid-4-O- $\beta$-D-allopyranoside (61), a new compound isolated from A. tanguticum, possesses a cis double bond. The glycosyl derivatives are generally monosaccharides composed of glucose, with only a small amount of gulose or allose, such as the new compounds 77 and $78 .{ }^{42}$ In addition to phenylpropionic acids, four phenylpropanols $(\mathbf{6 8}, \mathbf{7 0}-\mathbf{7 2})$ have also been isolated from A. tanguticum. ${ }^{43}$

There are few reports on the lignan and coumarin components of Aconitum plants. Four furofuran-type lignans, namely (+)-1-hydroxy-pinoresinol (85) from A. gymnandrum, ${ }^{49}$ phillygenin (86) from A. stapfianum, ${ }^{45}(+)$-pinoresinol (87) from $A$. tanguticum, ${ }^{47}$ and $(+)$-medioresinol (88) from A. kongboense, ${ }^{46}$ have been obtained to date (Fig. 3). In addition, a new coumarin derivative, 6,7-dimethoxy-8-hydroxy-3-[ $\beta$-( $p$-hydroxyphenyl) ethyl]-3,4-dihydroisocoumarin (84), was obtained from $A$. gymnandrum. ${ }^{49}$

Several phenylpropanoids have displayed anti-inflammatory properties. Caffeic acid (56) and its derivatives 57-59 from $A$. koreanum inhibited NO production in LPS-stimulated RAW 264.7 mouse macrophages $\left(\mathrm{IC}_{50}\right.$ values of $2.07,1.20,0.76$, and $2.37 \mu \mathrm{M}$, respectively) significantly more effectively than was achieved by the positive control L-NMMA ( IC $_{50}$ value of $\left.7.83 \mu \mathrm{M}\right)$ 
<smiles>[R]C(=O)/C=C/c1cc([R2])c([R])c([R])c1</smiles>

\begin{tabular}{|c|c|c|c|c|}
\hline No & name & $R_{1}, R_{2}, R_{3}, R_{4}$ & plants & ref \\
\hline 56 & caffeic acid & $\mathrm{R}_{1}=\mathrm{R}_{2}=\mathrm{R}_{3}=\mathrm{OH}, \mathrm{R}_{4}=\mathrm{H}$ & A. koreanum & 44 \\
\hline 60 & ethyl caffeate & $\mathrm{R}_{1}=\mathrm{OEt}, \mathrm{R}_{2}=\mathrm{R}_{3}=\mathrm{OH}, \mathrm{R}_{4}=\mathrm{H}$ & A. kongboense & 46 \\
\hline $65^{*}$ & $\begin{array}{l}\text { 3,4-dimethoxy-trans-cinnamic acid- } \\
\text { 9-O-6-D-allopyranoside }\end{array}$ & $\mathrm{R}_{1}=O-B_{-\mathrm{D}}-\mathrm{All}, \mathrm{R}_{2}=\mathrm{OH}, \mathrm{R}_{3}=\mathrm{OMe}, \mathrm{R}_{4}=\mathrm{H}$ & A. tanguticum & 41 \\
\hline 66 & (E)-ferulic acid 4-O-6-D-allopyranoside & $\mathrm{R}_{1}=\mathrm{OH}, \mathrm{R}_{2}=\mathrm{OMe}, \mathrm{R}_{3}=\mathrm{O}-\mathrm{B}_{-\mathrm{D}}-\mathrm{Glu}, \mathrm{R}_{4}=\mathrm{H}$ & A. tanguticum & 43 \\
\hline 67 & (E)-sinapic acid-4-O-B-glucopyranoside & $\mathrm{R}_{1}=\mathrm{OH}, \mathrm{R}_{2}=\mathrm{R}_{4}=\mathrm{OMe}, \mathrm{R}_{3}=0-6-\mathrm{D}-\mathrm{Glu}$ & A. tanguticum & 43 \\
\hline 69 & (E)-ferulic acid-4-O-B-glucopysoside & $\mathrm{R}_{1}=\mathrm{OH}, \mathrm{R}_{2}=\mathrm{OMe}, \mathrm{R}_{3}=O-B-\mathrm{D}-\mathrm{All}, \mathrm{R}_{4}=\mathrm{H}$ & A. tanguticum & 43 \\
\hline 73 & $\begin{array}{l}\text { 4-O-B-o-glucopyranosyl-(E)-ferulic } \\
\text { acid methyl ester }\end{array}$ & $\mathrm{R}_{1}=\mathrm{R}_{2}=\mathrm{OMe}, \mathrm{R}_{3}=\mathrm{O}-\mathrm{-}_{-\mathrm{D}}-\mathrm{Gul}, \mathrm{R}_{4}=\mathrm{H}$ & A. tanguticum & 47 \\
\hline 74 & $\begin{array}{l}(2 E)-3-\left[4-\left(6-{ }_{-}-\text {glucopyranoside)- }\right.\right. \\
\text { phenyl]acrylic acid }\end{array}$ & $R_{1}=O H, R_{2}=R_{4}=H, R_{3}=O-6-D-G l u$ & A. flavum & 48 \\
\hline 75 & ferulic acid methyl ester & $\mathrm{R}_{1}=\mathrm{R}_{2}=\mathrm{OMe}, \mathrm{R}_{3}=\mathrm{OH}, \mathrm{R}_{4}=\mathrm{H}$ & A. flavum & 48 \\
\hline 76 & ferulic acid & $\mathrm{R}_{1}=\mathrm{R}_{3}=\mathrm{OH}, \mathrm{R}_{2}=\mathrm{OMe}, \mathrm{R}_{4}=\mathrm{H}$ & $\begin{array}{l}\text { A. flavum } \\
\text { A. gymnandrum }\end{array}$ & $\begin{array}{l}48 \\
49\end{array}$ \\
\hline $77^{*}$ & methyl-4-B-D-allopyranosyl-ferulate & $R_{1}=R_{2}=O M e, R_{3}=0-{ }_{-}-_{D}-A l l, R_{4}=H$ & A. carmichaelii & 42 \\
\hline 78* & methyl-4-6-D-gulopyranosyl-cinnamate & $\mathrm{R}_{1}=\mathrm{OMe}, \mathrm{R}_{2}=\mathrm{O}-6-\mathrm{D}-\mathrm{Gul}, \mathrm{R}_{3}=\mathrm{R}_{4}=\mathrm{H}$ & A. carmichaelii & 42 \\
\hline 79 & methyl 4-6-ס-glucopyranosylferulate & $\mathrm{R}_{1}=\mathrm{R}_{3}=\mathrm{OMe}, \mathrm{R}_{2}=\mathrm{O}-\mathrm{B}_{-\mathrm{D}}-\mathrm{All}, \mathrm{R}_{4}=\mathrm{H}$ & A. carmichaelii & 42 \\
\hline 80 & $(E)$-p-hydroxycinnamic acid & $\mathrm{R}_{1}=\mathrm{R}_{3}=\mathrm{OH}, \mathrm{R}_{2}=\mathrm{R}_{4}=\mathrm{H}$ & $\begin{array}{l}\text { A. stapfianum } \\
\text { A. carmichaelii }\end{array}$ & $\begin{array}{l}45 \\
50\end{array}$ \\
\hline
\end{tabular}

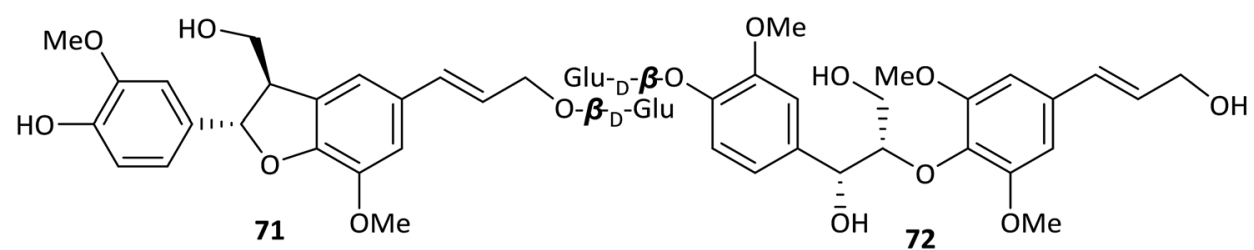

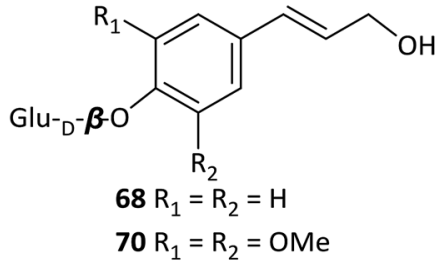<smiles>COc1cc([C@@H]2O[C@H]2CO)ccc1O</smiles>

\begin{tabular}{llll}
\hline No & name & plants & ref. \\
\hline $\mathbf{6 8}$ & syringin & A. tanguticum & 43 \\
$\mathbf{7 0}$ & $(E)-4-$-hydroxycinnamyl alcohol 4-O-B-D-glucopyranoside & A. tanguticum & 43 \\
$\mathbf{7 1}$ & $(7 S, 8 R)$-dehydrodiconiferyl alcohol-9'-O-B-D-glucopyranoside & A. tanguticum & 43 \\
$\mathbf{7 2}$ & citrusin B & A. tanguticum & 43 \\
$\mathbf{8 2}$ & epoxyconiferyl alcohol & A. stapfianum & 45 \\
$\mathbf{8 3}$ & dihydroconiferyl alcohol & A. gymnandrum & 49 \\
\hline
\end{tabular}

Fig. 2 (contd.)

by suppressing both the mRNA and protein expressions of iNOS and COX-2.44 New compound 78 from A. carmichaelii also inhibited NO production in LPS-stimulated RAW 264.7 cells with an $\mathrm{IC}_{50}$ value of $61.3 \mu \mathrm{g} \mathrm{ml^{-1 }} .^{42}$ Ferulic acid (76) inhibited TNF- $\alpha$ production in LPS-stimulated RAW 264.7 cells with an $\mathrm{IC}_{50}$ value of $27.6 \mu \mathrm{g} \mathrm{ml}^{-1} .^{47}$ The consistency of anti- inflammatory activities between the phenylpropanoids and DAs suggests that they all jointly contribute to the antiphlogistic effects of the medicinal Aconitum plants.

Besides, studies showed that phenylpropanoids also possess antineoplastic activities. Phenylpropionic acid 73 displayed selective cytotoxicity against gastric cancer MGC80 cells with an 


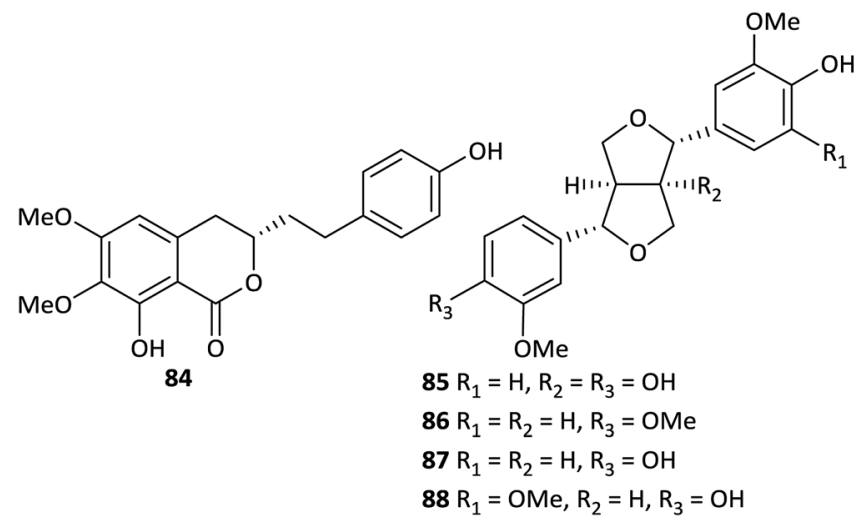

Fig. 3 Lignans and coumarins from Aconitum plants.

$\mathrm{IC}_{50}$ value of $38.1 \mu \mathrm{g} \mathrm{ml} \mathrm{m}^{-1}$, 9 and lignan 85 showed selective cytotoxicity against liver cancer $\mathrm{HepG} 2$ cells with an $\mathrm{IC}_{50}$ value of $48.7 \mu \mathrm{g} \mathrm{ml}^{-1}$. The Aconitum-derived drugs are also used in the treatment of various cancers. While a great many of DAs have been proved to possess certain cytotoxicity, ${ }^{10}$ these phenylpropanoids could also play a positive role in the antineoplastic effect of Acontim plants in conjunction with DAs.

\subsection{Other phenolics and acids}

In addition to the phenylpropionic acids described above, other types of phenolics and acids have been isolated from Aconitum plants as well. The first representative was aconitic acid (89) (Fig. 4), which was first found in A. napellus in the 19th century and named after the Aconitum genus. A number of studies have revealed that aconitic acid is not restricted to Aconitum plants but plays a vital role in the tricarboxylic acid cycle of aerobes. In addition, Jiang et al. reported the isolation of seven new aromatic acid derivatives (90-96) and five known analogues (97-

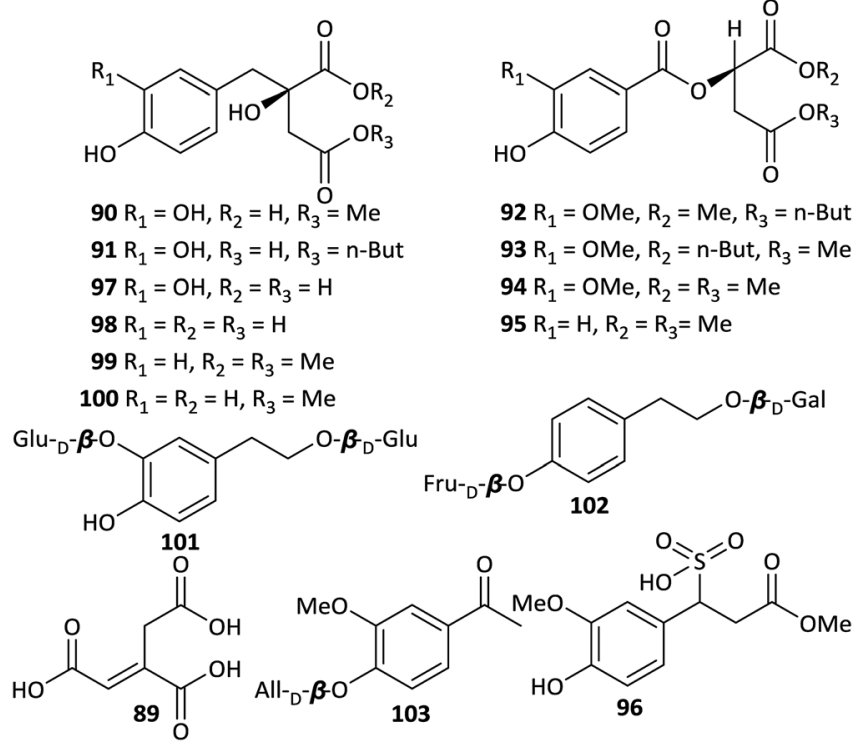

Fig. 4 Other phenolics and acids from Aconitum plants.
100) from the Fuzi. ${ }^{51}$ Compounds 90 and 91 are 2-benzylmalates (eucomate derivatives), 92-95 are 2-O-benzoylmalates, and $\mathbf{9 6}$ is a rare phenylpropionate containing a sulfonic acid group. The absolute configurations of eucomate derivatives were confirmed by X-ray crystallographic analysis of 4-methyl eucomate (100).

Many benzoic or phenylacetic acid derivatives with hydroxyl, oxygen alkyl, or glycosyl substituents have been obtained from Aconitum plants. Most of these compounds are common, structurally simple and widely distributed in the plant kingdom; new structures are rarely discovered. Only three new glycosides, namely, 2-(3-O- $\beta$-D-glucopyranosyl-4-hydroxyphenyl) ethanol 1-O- $\beta$-D-glucopyranoside (101), 2-(4-O- $\beta$-D-fructopyranosylphenyl)ethanol $\quad 1-O-\beta$-D-galactopyranoside (102), and 3-methoxy-4-O- $\beta$-D-allopyranosyl acetophenone (103), were isolated from A. tanguticum. ${ }^{47}$ Compounds 101 and 103 also possess anti-inflammatory activities, which inhibited TNF$\alpha$ production on LPS-stimulated RAW 264.7 macrophages with $\mathrm{IC}_{50}$ values of 38.2 and $27.6 \mu \mathrm{g} \mathrm{ml}{ }^{-1}$, respectively.

\subsection{Terpenoids and their glycosides}

In contrast to the wide variety of DAs present in Aconitum plants, terpenoids are rare. To the best of our knowledge, only three non-alkaloidal diterpenoids from the genus Aconitum have been reported, namely, atropurpuran (104) from A. hemsleyanum var. atropurpureum, ${ }^{11}$ atisenol (105) from A. heterophyllum, ${ }^{52}$ and Guan fu diterpenoid A (106) from A. coreanum (Fig. 5)..$^{53}$ All of them were isolated as new compounds. These non-alkaloidal diterpenoids are of great interest for their biogenetic pathway; they may be the earlier intermediates in the DA biogenesis. ${ }^{54}$ Notably, atropurpuran (104) is the first example of a diterpenoid with a novel 6/6/6/6/6 carbocyclic framework, which features an unprecedented cage-like skeleton containing an unusual tetracyclo[5.3.3. $\left.0^{4,9} \cdot 0^{4,12}\right]$-tridecane unit (rings B-E) fused to a highly functionalized cyclohexene fragment (ring A) with two adjacent chiral quaternary centres. Atropurpuran has drawn researchers' great attention for its intriguing structure, which has been introduced as "Hot off the Press" and synthesized successfully by outstanding chemists after report. ${ }^{55-57}$
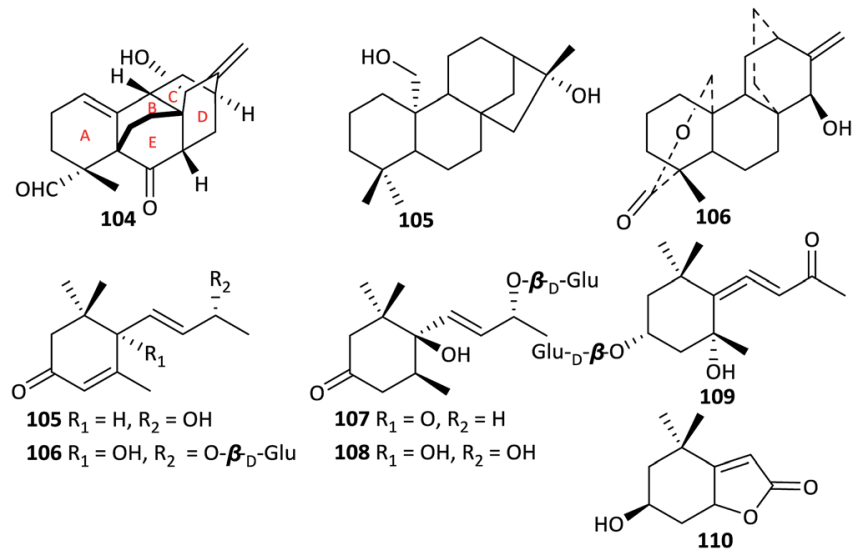

Fig. 5 Terpenoids from the Aconitum species. 


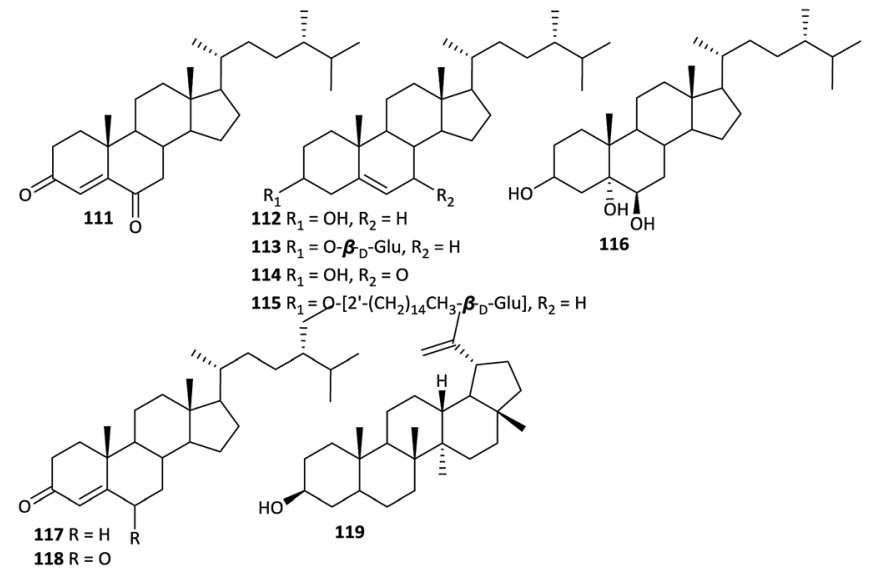

Fig. 6 Steroids from the Aconitum plants.

In addition, six megastigmane sesquiterpenoids and their glycosides, including (6R,7E,9R)-9-hydroxy-4,7-megastigmadien-3-one (105) from $A$. gymnandrum, ${ }^{49}$ vomifoliol- $O-\beta$-D-glucopyranoside (106), dihydrovomifoliol- $O$ - $\beta$-D-glucopyranoside (107), icariside $\mathrm{B}_{1}(\mathbf{1 0 8}),(3 R, 5 S, 6 S, 7 E, 9 R)$-megastig-man-7-ene3,5,6,9-tetrol-9- $O$ - $\beta$-D-glucopy-ranoside (109) and loliolide (110) from $A$. tanguticum, ${ }^{39,43}$ have been reported. These compounds mainly vary in the degree of oxidation of their cyclohexene ring and the number and type of substituents on the side chain.

\subsection{Steroids}

While little attention has been paid to the steroids in Aconitum plants, approximately nine steroids have been reported from various Aconitum plants (Fig. 6). Most of these steroids are common phytosterols, including $\beta$-sitosterol (112), daucosterol (113), 7-oxo- $\beta$-sitosterol (114), $\beta$-sitosteryl-3- $O$ - $\beta$-D-glucopyranoside-2'-O-palmitate (115), sitostanetriol (116), $\beta$-sitost-4-en-3one (117), 22-dihydro-stigmast-4-en-3,6-dione (118) and lupeol (119),,$^{31,45,58}$ which are common in many plants. $24 S$-ergost-4-en3,6-dione (111) is the only new steroid isolated from Aconitum plants. ${ }^{59}$

\subsection{Free fatty acids (FFAs)}

Fatty acids have been attracting increased interest from the food and pharmacy industries due to their nutritional and biological importance. But until now, there have been few studies on the FFA components of Aconitum plants, and these studies were performed using GC-MS techniques. A GC-MS-based analysis of the FFAs in A. taipeicum performed by Yue et al. revealed that the major FFAs in this plant are linoleic acid (120, 46.24\%), palmitic acid $(\mathbf{1 2 1}, 28.24 \%)$, and oleic acid $(\mathbf{1 2 2}, 15.93 \%)$, followed by stearic acid $(\mathbf{1 2 3}, 4.35 \%)$, linolenic acid $(\mathbf{1 2 4}, 2.87 \%)$, and palmitoleic acid (125, 2.38\%) (Fig. 7). ${ }^{60}$ Similar results were found in $A$. carmichaelii, in which linoleic acid (35.97\%), palmitic acid (14.24\%), oleic acid (7.42\%) and linolenic acid (7.29\%) are the major FFA components. ${ }^{61}$ In addition, Qu et al. reported that $A$. szechenyianum is remarkably rich in linoleic acid $(\mathbf{1 2 4}, 41.32 \%)$, palmitic acid (121, 29.64\%) and linolenic acid (120, 23.77\%). ${ }^{62}$ These FFAs could be bonded to $\mathrm{C}_{19}$-DAs through ester exchange

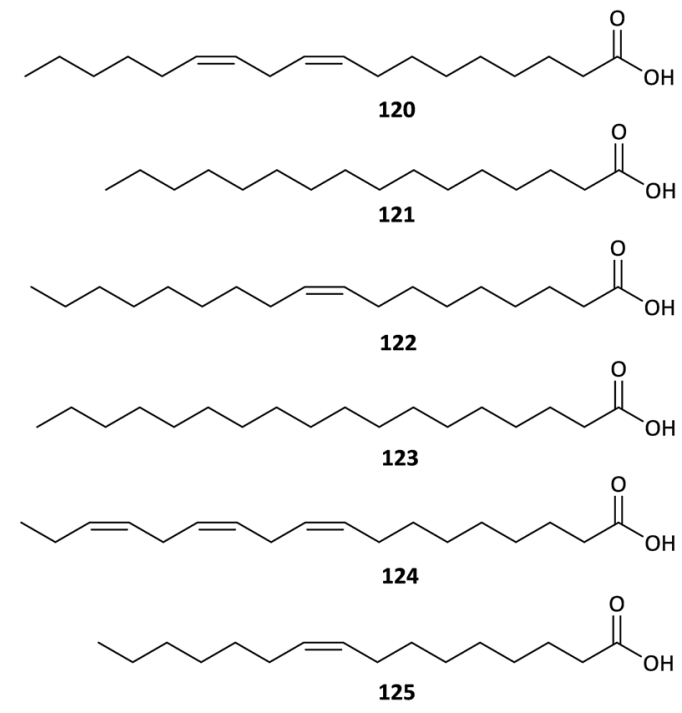

Fig. 7 Main FFAs in Aconitum plants.

reaction to generate lipo-alkaloids, a kind of DAs consist of a $\mathrm{C}_{19}$-DAs skeleton and one or two fatty acid side chains substituted at $\mathrm{C}-8$ or $\mathrm{C}-14$, which have been reported to exhibited impressive bioactivities. ${ }^{\mathbf{1 0 , 6 3}}$

\subsection{Polysaccharides}

The discovery of bioactive homogeneous polysaccharides from Aconitum plants occurred much later than the identification of the small-molecule compounds described above due to the difficulties associated with both purification and identification of polysaccharides. In 1985, Chohachi et al. isolated the first four homogeneous polysaccharides, named aconitans A-D, from Fuzi using an activity-guided separation strategy, and the compounds showed strong hypoglycaemic effects in mice. However, their structures have not been clarified. ${ }^{64}$ Later, in 2006, Zhao et al. separated a water-soluble homogeneous polysaccharide, FPS-1, from the same herb, and the compound was determined to be an $\alpha$ - $(1 \rightarrow 6)$-D-glucan with a weight-average molecular weight (m.w.) of approximately 14000 Da. This glucan is highly branched with a single glucose at C-3 of every fourth residue along the main chain. FPS-1 displays various biological activities. In immunopharmacological studies, FPS-1 stimulated murine lymphocyte proliferation induced by concanavalin A or LPS in a potent manner both in vitro and in vivo in addition to stimulating splenocyte antibody production, giving this compound potential as a natural immunomodulator. ${ }^{65}$ FPS-1 also produces antidepressant-like and neurogenic effects with rapid onset in mice. ${ }^{66}$ In addition, FPS-1 has been shown to increase autophagy activity to protect against starvation-induced cytotoxicity in H9c2 cells through the AMPK/ mTOR pathway activation, which could be exploited for drug discovery related to coronary heart disease and heart failure. ${ }^{67}$

Gao et al. reported a hot alkali extracted polysaccharide, AKP, from another important Aconitum herb Caowu, which was determined to be a linear $\alpha$-(1 $\rightarrow 3),(1 \rightarrow 4)$-D-glucan with a m.w. of approximately $14000 \mathrm{Da}$, and the $(1 \rightarrow 3)$-linked and $(1 \rightarrow 4)$ - 
linked $\alpha$-D-Glup residues were in a ratio of $1: 7 .{ }^{68}$ AKP had significant in vitro antioxidant activities; it showed a high FRAP value and noticeable effects on the scavenging of free radicals and chelating ferrous ions.

Several studies have evaluated the bioactive polysaccharides present in Korean monkshood (A. coreanum), which is also called Guanbaifu in TCM. The homogeneous polysaccharide KMPS-2A consisted of a $[\alpha-1,6-\mathrm{D}-\mathrm{Glc}]_{n}$ repeating structure with a m.w. of $676000 \mathrm{Da}$, and its sulfated derivatives exhibited better anticomplementary activity than heparin, which indicated its potential as a complement inhibitor. ${ }^{69}$ Another polysaccharide, KMPS-2E, consisted of $[\rightarrow 6)-\beta$-D-Galp $(1 \rightarrow 3)-\beta-\mathrm{L}^{-}$ Rhap-( $1 \rightarrow 4)-\beta$-D-GalpA- $(1 \rightarrow 3)-\beta$-D-Galp- $(1 \rightarrow]$ units with $\rightarrow 5)-\beta$ $\mathrm{D}$-Arap $(1 \rightarrow 3,5)-\beta$-D-Arap $(1 \rightarrow$ side chains attached to the backbone through $\mathrm{O}-4$ of $(1 \rightarrow 3,4)$-L-Rhap. T- $\beta$-D-Galp is attached to the backbone through $0-6$ of the $(1 \rightarrow 3,6)-\beta$-D-Galp residues, and $\mathrm{T}-\beta$-D-Ara is connected to the end group of each chain. ${ }^{70}$ KMPS-2E showed significant anti-inflammatory effects both in LPS-stimulated RAW 264.7 macrophages and carrageenaninduced hind paw oedema models. KMPS-2E could inhibit the expression of iNOS and inflammatory cytokines mediated by the NF-kB signalling pathways in macrophages. Moreover, KMPS$2 \mathrm{E}$ has no cytotoxicity up to $200 \mathrm{mg} \mathrm{ml}^{-1}$, which highlights its potential in the development of novel, natural antiinflammatory agents.

Plant polysaccharides are well-known for their biocompatible and non-toxic properties, which have been applied to clinical side and the market of health products widely. As summarized above, polysaccharides from Aconitum plants have displayed broad bioactivities, including antioxidant, antitumour, antiphlogistic and immunoregulatory activities, as well as beneficial effects on the cardio-cerebrovascular system. Hence, polysaccharides from Aconitum plants may be considered to serve as a new individual effective part in the application of functional foods and drugs.

\section{Conclusions}

Aconitum is an important genus that is widely used in TCM and other Asian medical traditions. The data summarized above revealed that there are abundant non-alkaloidal components, including flavonoids, phenylpropanoids, phenolic acids, terpenoids, steroids, FFAs and polysaccharides, with broad bioactivities in Aconitum plants. In view of the chemical diversity described, the major classes of non-alkaloidal constituents found in the Aconitum plants were flavonoids, followed by phenylpropanoids and other phenolic acids. Among these compounds, several are new compounds, and their various biological activities have been reported. These findings underscore the large chemical and biological diversity of nonalkaloidal constituents in Aconitum plants, which could not only serve as a vast resource for drug discovery separately, but also contribute to the therapeutic effects of Aconitum-derived drugs.

Although phytochemical and biological studies on nonalkaloidal constituents of Aconitum species have received considerable interest, some deficiencies remain. Firstly, most of the current studies are still aimed at illuminating the alkaloids in Aconitum species and not at characterizing the non-alkaloidal constituents. And investigations of non-alkaloidal constituents are restricted to the widespread Aconitum species, such as $A$. carmichaelii, $A$. tanguticum in China, while most less-common species are still largely unexplored. The potential role of nonalkaloidal constituents in the pharmacological activities of Aconitum plants remains ignored, as well as their chemotaxonomical significance. An extensive investigation of other species, especially those species used medicinally, is necessary. Secondly, various biological activities of the extracts and pure compounds were mainly investigated by using in vitro tests and less were carried out by in vivo models. Therefore, there are few reported data focused on toxicity, side effects, and clinical efficiency of these non-alkaloidal constituents. The few pharmacological studies are still insufficient to validate the effects of the non-alkaloidal constituents in Aconitum species, which hinders its application and promotion. It is necessary to evaluate the biological activities of the non-alkaloidal constituents using both in vitro and in vivo models.

\section{Conflicts of interest}

There are no conflicts to declare.

\section{Acknowledgements}

This work was financially supported by a grant from the National Natural Science Foundation of China (No. 31860095) and a grant from Guizhou Science and Technology Foundation of China (No. QKHJC[2018]1193).

\section{References}

1 L. Q. Li and K. Yuichi, Flora of China, 2001, vol. 6, pp. 149222.

2 G. Zhou, L. Tang, X. Zhou, T. Wang, Z. Kou and Z. Wang, J. Ethnopharmacol., 2015, 160, 173-193.

3 E. Nyirimigabo, Y. Xu, Y. Li, Y. Wang, K. Agyemang and Y. Zhang, J. Pharm. Pharmacol., 2015, 67, 1-19.

4 P. G. Xiao, F. P. Wang, F. Gao, L. P. Yan, D. L. Chen and Y. Liu, Acta Phytotaxon. Sin., 2006, 44, 1-46.

5 F. P. Wang, Q. H. Chen and X. Y. Liu, Nat. Prod. Rep., 2009, 27, 529-570.

6 Q. Guo, H. Xia, G. Shi, T. Zhang and J. Shi, Org. Lett., 2018, 20, 816-819.

7 N. H. Chen, Y. B. Zhang, W. Li, P. Li, L. F. Chen, Y. L. Li, G. Q. Li and G. C. Wang, RSC Adv., 2017, 7, 24129-24132.

8 Y. Shu, T. P. Yin, J. P. Wang, D. Gan, Q. Y. Zhang, L. Cai and Z. T. Ding, Chin. J. Nat. Med., 2018, 16, 866-870.

9 M. Reina and A. González-Coloma, Phytochem. Rev., 2007, 6, 81-95.

10 X. X. Liang, Y. Y. Gao and S. X. Luan, RSC Adv., 2018, 8, 23937-23946.

11 P. Tang, Q. H. Chen and F. P. Wang, Tetrahedron Lett., 2009, 50, 460-462. 
12 I. I. Samokish, A. L. Shinkarenko and V. A. Makarov, Chem. Nat. Compd., 1969, 5, 364-365.

13 V. D. Loi, B. T. Tung, N. T. Hai and N. T. Vung, J. Chem. Pharm. Res., 2015, 7, 228-234.

14 B. B. Shrestha, S. Dall'Acqua, M. B. Gewali, P. K. Jha and G. Innocenti, Carbohydr. Res., 2006, 341, 2161-2165.

15 C. Mariani, A. Braca, S. Vitalini, N. De Tommasi, F. Visioli and G. Fico, Phytochemistry, 2008, 69, 1220-1226.

16 L. Xu, X. Zhang, L. M. Lin, C. Li, Z. M. Wang and Y. M. Luo, J. Asian Nat. Prod. Res., 2013, 15, 737-742.

17 N. N. Pogodaeva, S. V. Fedorov, L. V. Kanitskaya and A. A. Semenov, Russ. Chem. Bull., 2000, 49, 1905-1909.

18 A. Braca, G. Fico, I. Morelli, F. De Simone, F. Tomè and N. De Tommasi, J. Ethnopharmacol., 2003, 86, 63-67.

19 S. Vitalini, A. Braca, D. Passarella and G. Fico, Fitoterapia, 2010, 81, 940-947.

20 J. M. He, T. P. Yin, Y. Chen, L. Cai, Z. G. Tai, Z. J. Li, C. S. Liu, Y. R. Wang and Z. T. Ding, J. Funct. Foods, 2015, 17, 371-379.

21 T. P. Yin, L. Cai, Y. Xing, J. Yu, X. J. Li, R. F. Mei and Z. T. Ding, J. Asian Nat. Prod. Res., 2016, 18, 603-610.

22 H. Ahmad, S. Ahmad, S. A. A. Shah, A. Latif, M. Ali, F. A. Khan, M. N. Tahir, F. Shaheen, A. Wadood and M. Ahmad, Bioorg. Med. Chem., 2017, 25, 3368-3376.

23 C. Marín, J. G. Díaz, D. Irure Maiques, I. Ramírez-Macías, M. J. Rosales, R. Guitierrez-Sánchez, R. Cañas and M. Sánchez-Moreno, Phytochem. Lett., 2017, 19, 196-209.

24 F. Gelsomina, S. Alberto, B. Alessandra, A. Elisabetta, M. Ivano and T. Franca, Biochem. Syst. Ecol., 2003, 31, 293301.

25 J. G. Díaz, J. G. Ruiz, B. R. Días, J. A. G. Sazatornil and W. Herz, Biochem. Syst. Ecol., 2005, 33, 201-205.

26 C. E. Lim, J. H. Park and C. W. Park, Plant Syst. Evol., 1999, 218, 125-131.

27 G. Fico, A. Braca, I. Morelli and F. Tomè, Fitoterapia, 2003, 74, 420-422.

28 I. I. Samokish, Chem. Nat. Compd., 1974, 10, 809.

29 T. Zhapova, L. D. Modonova, N. N. Pogodaeva, A. L. Vereshchagin, A. G. Gorshkov, S. V. Zinchenko and A. A. Semenov, Chem. Nat. Compd., 1992, 28, 421-429.

30 W. K. Whang, I. S. Oh, M. T. Lee and I. H. Kim, Korean J. Pharmacogn., 1994, 25, 336-341.

31 D. K. Kim, J. H. Kwak, K. W. Song, H. C. Kwon, O. P. Zee and K. R. Lee, Korean J. Pharmacogn., 1996, 27, 75-79.

32 H. J. Jeong, W. K. Whang and I. H. Kim, Planta Med., 1997, 63, 329-334.

33 G. Fico, A. Braca, A. R. Bilia, F. Tomè and I. Morelli, J. Nat. Prod., 2000, 63, 1563-1565.

34 S. Vitalini, A. Braca and G. Fico, Phytochem. Lett., 2012, 5, 476-479.

35 G. Fico, A. Braca, N. De Tommasi, F. Tomè and I. Morelli, Phytochemistry, 2001, 57, 543-546.

36 G. Fico, A. Braca, A. R. Bilia, F. Tomè and I. Morelli, Planta Med., 2001, 67, 287-290.

37 S. Dall'Acqua, B. B. Shrestha, M. B. Gewali, P. K. Jha, M. Carrara and G. Innocenti, Nat. Prod. Commun., 2008, 3, 1985-1989.
38 J. C. Luis, F. Valdes, R. Martin, A. J. Carmona and J. G. Diaz, Fitoterapia, 2006, 77, 469-471.

39 M. Luo, L. M. Lin, C. Li, Z. M. Wang and W. B. Guo, China J. Chin. Mater. Med., 2012, 37, 1245-1248.

40 L. Xu, M. Luo, L. M. Lin, X. Zhang, C. Li, Z. M. Wang and Y. M. Luo, J. Asian Nat. Prod. Res., 2013, 15, 743-749.

41 Y. R. Li, L. Xu, C. Li, Z. M. Wang and L. X. Yang, J. Asian Nat. Prod. Res., 2014, 16, 730-734.

42 T. Q. Do, B. N. Truong, H. D. T. Mai, T. L. Nguyen, V. H. Nguyen, H. D. Nguyen, T. D. Nguyen, T. C. Nguyen, T. V. Luong, L. T. Giang, V. M. Chau and V. C. Pham, J. Asian Nat. Prod. Res., 2018, 1-9.

43 L. Xu, Y. R. Li, C. Li, L. M. Lin, Z. M. Wang and Y. M. Luo, China J. Chin. Mater. Med., 2013, 38, 2018-2825.

44 K. H. Park, M. Park, S. E. Choi, M. S. Jeong, J. H. Kwon, M. H. Oh and M. W. Lee, Biol. Pharm. Bull., 2009, 32, 2029-2033.

45 T. P. Yin, Y. Chen, Y. Shu, L. Cai and Z. T. Ding, Yunnan Daxue Xuebao, 2018, 40, 148-153.

46 J. J. Fu, J. J. Qin, Q. Zeng, H. Z. Jin and W. D. Zhang, Chem. Nat. Compd., 2011, 47, 854-855.

47 Y. R. Li, T. Liu, R. Y. Yan, L. Q. Hui, L. M. Lin, C. Y. Cao, S. S. Guo, L. X. Yang, W. H. Feng, C. Li and Z. M. Wang, Phytochem. Lett., 2015, 11, 311-315.

48 X. F. Wang, L. Yu, W. J. Hao, L. Ma, L. Yin and X. Y. Fu, Chem. Nat. Compd., 2016, 52, 769-770.

49 J. Q. Zhao, Y. M. Wang, S. Wang, J. Dang, Y. P. Shi, L. J. Mei and Y. D. Tao, Nat. Prod. Res., 2016, 30, 1746-1752.

50 J. Zhang, G. B. Sun, Q. F. Lei, G. Z. Li, J. C. Wang and J. Y. Si, Acta Pharmacol. Sin., 2014, 49, 1150-1154.

51 Z. B. Jiang, B. Y. Jiang, C. G. Zhu, Q. L. Guo, Y. Peng, X. L. Wang, S. Lin and J. G. Shi, J. Asian Nat. Prod. Res., 2014, 16, 891-900.

52 S. W. Pelletier, A. M. M. Ateya, J. Finer-Moore, N. V. Mody and L. C. Schramm, J. Nat. Prod., 1982, 45, 779-781.

53 C. H. Yang, X. C. Wang, Q. F. Tang, W. Y. Liu and J. H. Liu, Helv. Chim. Acta, 2008, 91, 759-765.

54 M. Weber, K. Owens and R. Sarpong, Tetrahedron Lett., 2015, 56, 3600-3603.

55 A. H. Robert and A. Sutherland, Nat. Prod. Rep., 2009, 26, 151.

56 J. Gong, H. Chen, X. Y. Liu, Z. X. Wang, W. Nie and Y. Qin, Nat. Commun., 2016, 7, 12183.

57 T. Suzuki, A. Sasaki, N. Egashira and S. Kobayashi, Angew. Chem., Int. Ed. Engl., 2011, 50, 9177-9179.

58 H. Y. Wang, A. X. Zuo, Y. Sun and G. X. Rao, J. Chin. Med. Mater., 2014, 37, 1391-1395.

59 K. A. Eshbakova, B. Tashkhodzhaev, Z. I. Tursunov, K. K. Turgunov, K. M. Bobakulov and N. D. Abdullaev, Chem. Nat. Compd., 2011, 47, 73-75.

60 X. F. Yue, Y. N. Zhang, J. Zhang and Z. Q. Zhang, Anal. Methods, 2010, 2, 668.

61 Y. Liang, G. Y. Yan, J. L. Wu, X. Zong, Z. Liu, H. Zhou, L. Liu and N. Li, Phytochem. Anal., 2018, 29, 398-405.

62 W. X. Qu, Z. L. Mou, H. Y. Cui and Z. Q. Zhang, Phytochem. Anal., 2011, 22, 199-204. 
63 T. Kiss, B. Borcsa, P. Orvos, L. Tálosi, J. Hohmann and D. Csupor, Planta Med., 2017, 83, 1321-1328.

64 C. Konno, M. Murayama, K. Sugiyama, M. Arai, M. Murakami, M. Takahashi and H. Hikino, Planta Med., 1985, 51, 160-161.

65 C. Zhao, M. Li, Y. Luo and W. Wu, Carbohydr. Res., 2006, 341, 485-491.

66 H. C. Yan, H. D. Qu, L. R. Sun, S. J. Li, X. Cao, Y. Y. Fang, W. Jie, J. C. Bean, W. K. Wu, X. H. Zhu and T. M. Gao, Int. J. Neuropsychopharmacol., 2010, 13, 623-633.
67 L. Z. Liao, Y. L. Chen, L. H. Lu, Y. H. Zhao, H. L. Guo and W. K. Wu, Am. J. Chin. Med., 2013, 41, 353-367.

68 T. Gao, H. Bi, S. Ma and J. Lu, Int. J. Biol. Macromol., 2010, 46, 85-90.

69 X. J. Li, J. Y. Jiang, S. S. Shi, Y. Li, Y. B. Jiang, Y. Ke and S. C. Wang, Chem. J. Chin. Univ., 2014, 35, 1423-1426.

70 X. J. Li, J. Y. Jiang, S. S. Shi, S. W. A. Bligh, Y. Li, Y. B. Jiang, D. Huang, Y. Ke and S. C. Wang, PLoS One, 2014, 9, e99697. 